\title{
Commonplace
}

\section{Library Comprehensive Content Strategy}

Peter Barr

Published on: Mar 02, 2021

License: Creative Commons Attribution 4.0 International License (CC-BY 4.0). 


\section{Feedback}

The University of Sheffield Library welcomes feedback on this draft of our proposed Comprehensive Content Strategy. We are currently consulting with our user communities for their feedback on the proposals but we would welcome input from interested parties beyond the institution.

The intention is to move away from thinking about how wider issues impact upon the 'collection', but rather to think about collections management as part of an interrelated set of activities that connect our users with content. From fellow Library and Information practitioners we are seeking constructive criticism into the practical workability of our proposals, and the extent to which this will facilitate a transformational shift in our approach.

Please use the annotation and commenting features here on Commonplace. Alternatively if you wish to discuss further, then please contact Peter Barr, Head of Content \& Collections (p.barr@sheffield.ac.uk).

\section{Purpose}

This strategy governs how the University Library directs activities to serve our user communities. It supports the University's digital education goals and the aim of developing an equitable and open research environment.

It establishes how the Library:

- provides access to the content required for teaching and research at the University

- builds, manages and shares collections of ongoing cultural value and showcasing the University's research

- transforms academic publishing and scholarly communication

\section{Principles}

The Library will acquire, manage and develop content in line with the values of the university based upon these core principles

- User-centred - we will seek to understand the needs and viewpoints of our communities, and conduct activities in keeping with them. We will collect the evidence that allows us to better understand this. 
- Transparent - we will be open in our activities and decision-making. Where principles come into conflict the decision-making will be openly explained.

- Open - we will strive for and facilitate the development of an Open Research environment.

- Sustainable - we will always seek to achieve the best value for money, without supporting activities that cause undue environmental impact.

- Inclusive - we will recognise the historical and active biases born of a lack of diversity across the Library workforce and wider University community, we will invest our resources to include those missing voices in our collections and wider work.

- Ethical - we will be socially responsible, and take no actions that reinforce existing inequalities.

\section{Supported Communities}

The core communities of the Library are the teachers, researchers and students of the University. As a major research library the collections will inspire scholarly research beyond the institution. The Library will also support alumni of the University and the wider communities of the city and region of Sheffield.

These communities are not homogeneous and consist of individuals from diverse disciplinary, social and cultural backgrounds. The Library will recognise this difference in how it supplies, collects and manages content.

\section{Partnerships}

To deliver our service requirements the Library works closely with commercial publishers and vendors, while accepting the limits of these relationships.

To achieve our strategic objectives, in support of digital education and open research, we work in partnership with sector partners, consortia, research funders and community groups.

\section{Access to Content}

The Library will enable access to scholarly and bibliographic content for our user communities, in ways that are accessible, sustainable and legal. Access to this content is essential for both teaching and learning, and the research of the University. We also recognise the profound pedagogical and social role of reading. We will provide access to peer-reviewed and wider academic literature, and to primary source material to 
allow for original study. We will seek to allow for all forms of reading and analysis, human and computational.

Access will be provided in the following ways:

- Open Access - the preferred method to meet the commitment to provide access to content is to allow for ethical, sustainable, open access for all.

- Digital - the assumption will be that, unless superseded by pedagogical, academic or accessibility imperatives, content will be delivered electronically.

- Paid Subscription - the Library will subscribe to commercial content where it is not possible to make it open access, preferring content and business models that do not perpetuate restrictive monopolies and high profits within academic publishing.

- Ownership - the Library will seek ownership of content only for the development of its collections, or where this is the more economical method to supply content to its communities.

- Infrastructure - the Library will fund and support infrastructure that enables access to content, and undertake the work to make our content accessible.

The Library recognises that it cannot claim to be neutral with regard to the content it provides. We will strive to balance the views of our user communities with regard to contestable and controversial content. We believe in freedom of expression but this does not supersede our commitment to a safe and inclusive environment for all.

\section{Collections}

The University Library possesses and manages collections that have been built over the history of the institution. They exist to be used as widely as possible, and to support the mission of the University. The value of these collections is derived in a number of ways:

- Academic - the Library collections provide a record of academic research. The dissemination of findings, and the scrutiny of data and source material, underpins the ability of this research to impact society.

- Cultural - the Library collections represent a part of the intellectual heritage of the world. They have an independent worth beyond the operational needs of the University.

- Symbolic - what is kept, and how it is kept is a powerful symbol of what is considered intellectually valuable. Consequently, the Library's collections contain an underlying message that must be inclusive and enabling for the wider goals of the University. 
- Organisational \& Curatorial - the organisation, description and presentation of the collections is meaningful and has worth alongside the content itself.

- Conservational - as part of a wider network of academic libraries and cultural organisations, the Library is committed to protect access to content for posterity.

- Operational - collections are means to provide access to content to user communities (as outlined above).

- Financial - the Library collections represent a significant financial commitment over the course of many years and as such we must acquire, maintain and dispose of material responsibly.

Different collections will deliver different value. This value is demonstrated by specific relevance for supported user communities. The Library will prioritise the building and holding of collections that are unique, relevant and curated to support the teaching and research of the University, and to showcase this research to the wider world.

\section{Use of Influence}

The University of Sheffield operates a major research library that is in possession of world leading collections and a multi-million pound budget. As such there is a responsibility to use this position to advocate for wider objectives within research and teaching.

The Library supports the University Digital Education Strategy and the creation of an open research culture adhering to FAIR (findable, accessible, interoperable, reusable) principles[1]. We must consider the role libraries play in the publishing process beyond the purchase of content and the maintenance of collections. With the academic departments and other professional services of the University, and our sector partners, the Library plays a significant role in the transformation of scholarly communication. The Library must also build our collections inclusively and in collaboration with the efforts of other libraries and archives around the world.

This commitment is based upon dialogue with user communities to enable individuals to better advocate for their own rights within academic publishing. The Library is opposed to restrictive practices and the pursuit of high profits within academic publishing. We will support initiatives beyond the library that value the dissemination of knowledge above the pursuit of commercial profit by the commitment of staffing, resources and budgets. The Library will understand the wider cultural landscape and ensure the content we provide access to is in alignment with a fair and equitable settlement for all scholars. 


\section{Citations}

1. https://www.go-fair.org/fair-principles/ $\triangleq$ 